\title{
Investigation of the Effects of Some Physical Parameters and Hall Current on MHD Fluid Flow with Heat Flux over a Porous Medium
}

\author{
Muhammad Nasir Sarki', Abdullahi Ahmed², Ime Jimmy Uwanta ${ }^{3}$ \\ ${ }^{1}$ Department of Mathematics, Kebbi State University of Science and Technology, Aliero, Nigeria \\ ${ }^{2}$ Department of Mathematics, Kebbi State Polytechnic, Dakingari, Nigeria \\ ${ }^{3}$ Department of Mathematics, Usmanu Danfodiyo University, Sokoto, Nigeria \\ Email: nasirsarki3@gmail.com, abdahmej@gmail.com,imeuwanta@yahoo.com
}

How to cite this paper: Sarki, M.N., Ahmed, A. and Uwanta, I.J. (2021) Investigation of the Effects of Some Physical Parameters and Hall Current on MHD Fluid Flow with Heat Flux over a Porous Medium. Advances in Pure Mathematics, 11, 652-664.

https://doi.org/10.4236/apm.2021.117043

Received: December 2, 2020

Accepted: July 3, 2021

Published: July 6, 202

Copyright $\odot 2021$ by author(s) and Scientific Research Publishing Inc.

This work is licensed under the Creative Commons Attribution-NonCommercial International License (CC BY-NC 4.0).

http://creativecommons.org/licenses/by-nc/4.0/

\begin{abstract}
In this paper, an investigation of the effects of some physical parameters and Hall current on magneto hydrodynamics (MHD) fluid flow with heat flux over a porous medium was carefully examined, taking into consideration Hall effects where the temperature and concentration are assumed to be oscillating with time. Furthermore, perturbation method is used in solving the governing equations. The profiles of velocity, temperature and concentration are presented graphically, going into the problem the primary and secondary velocity are presented and compute for some physical parameters such as mass Grashof number $(G c)$, Schmidt number $S c$, Prandtl number $(P r)$ viscoelastic parameter $\left(K_{1}\right)$ and hall current parameter $(m)$. Results indicated that primary velocity increases with increase in values of $G c$ on one hand and on the other hand it decreases with increase in the values of $P r, K_{1}$ and $m$. Secondary velocity demonstrated opposite trend.
\end{abstract}

\section{Keywords}

Hall Current, Heat Flux, Porous Medium

\section{Introduction}

The problems of fluid flow continue to attract the attention of so many researchers in engineering science, applied mathematics and applied geophysics. Heat and mass transfer is used in aerodynamic, extrusion of plastic sheets and other engineering processes which include chemical engineering and petroleum engineering [1]. Flows through porous media are very much prevalent in nature 
and therefore the study of flows through porous medium has become important to many scientists. In view of the above [2], studied MHD free and forced convection flow of Newtonian fluid through a porous medium in an infinite vertical plate in the presence of thermal radiation, heat transfer and surface temperature oscillation. [3] examined heat and mass transfer in MHD viscous elastic fluid flow through a porous medium over a stretching sheet with chemical reaction. In the last six years many investigations dealing with heat flow problem over a vertical porous plate with variable suction, soret effects, heat absorption, dissipation and have been investigated by so many researchers. Hall current effects on unsteady magnetohydrodynamics flow between streaching sheet and an oscillating porous upper parallel plate with constant suction [4]. Mass Transfer and Free Convection Effects on the Flow Past an Accelerated Vertical Plate with Variable Suction or Injection, was illustrated by [5]. [6] studied radiation effects on heat and mass transfer over a vertical plate with Newtonian fluid. Hall current effects on unsteady Mhd fluid flow with radiative heat flux and heat source over a porous medium, was analytically examined by [7]. [8] conducted Analytical study of MHD radiation-convection with surface temperature oscillation and secondary flow effects. [9] have investigated the effects of hall current on the steady hydromagnetic flow in an incompressible viscous fluid. [2] studied steady and unsteady magneto hydrodynamic (MHD) viscous, incompressible free and forced convective flow of an electrically conducting Newtonian fluid through a porous medium in the presence of appreciable thermal radiation heat transfer and surface temperature oscillation taking hall current into account. In addition [10] analyzed MHD free convection and mass transfer flows with hall current, viscous dissipation joul heating and thermal diffusion. [11] analyzed MHD free convection and mass transfer flow in a porous media with simultaneous rotating fluid. [12] had discussed the MHD unsteady free convective Walter's memory flow with constant suction and heat sink. [13] investigated radiation effects on heat and mass transfer over an exponentially accelerated infinite vertical plate with chemical reaction. Combined influence of radiation absorption and hall current effects on MHD double diffusive free convective flow past a stretching sheet, was demonstrated [14]. [15] analyzed hall current effects on MHD free convective heat and mass transfer flow past an oscillating vertical porous plate in the presence of radiation. Effects of radiation, chemical reaction and porosity of the medium on MHD flow past an oscillating plate with heat and mass transfer was examined by [16]. [17] studied Hall current effects on unsteady magnetohydrodynamics flow between stretching sheet and an oscillating porous upper parallel plate with constant suction. The effect of slip conditions and Hall current on unsteady MHD flow of a viscoelastic fluid past on infinite vertical porous plate through porous medium was studied by [18], the research investigated that primary velocity significantly decreases with the increases of Hall current parameter. Considering [18], in this research the effects of Hall current on MHD fluid flow are investigated to include radiative heat flux and concentration over a 
porous medium.

\section{Problem Formulation}

Consider the flow of incompressible memory fluid in an infinite plane with heat and mass transfer, under the influence of an induced magnetic field and constant suction. The $x$-axis is taken along the plane in the upward direction and a straight line perpendicular to that of the $y$-axis. All fluid properties are assumed constant. Since the fluid is conducting, the magnetic Reynolds number is much less than unity and hence the induced magnetic field is not neglected.

The equations governing the flow under Boussineqs approximation are:

Continuity equation:

$$
\frac{\partial v}{\partial y}=0
$$

Momentum equation

$$
\begin{aligned}
\frac{\partial u^{\prime}}{\partial t}+v_{0} \frac{\partial u^{\prime}}{\partial y^{\prime}}= & v \frac{\partial^{2} u^{\prime}}{\partial y^{2}}-v \frac{u^{\prime}}{K^{*}}+g \beta^{*}\left(C_{0}^{\prime}-C_{d}^{\prime}\right)+g \beta^{*}\left(T_{0}^{\prime}-T_{d}^{\prime}\right) \\
& -\frac{\sigma \beta_{0}^{2}}{\rho\left(1+m^{2}\right)}(u+m w)-K_{1}\left\{\frac{\partial^{3} u^{\prime}}{\partial t \partial y^{2}}\right\} \\
\frac{\partial w^{\prime}}{\partial t^{\prime}}+v_{0} \frac{\partial w^{\prime}}{\partial y^{\prime}}= & v \frac{\partial^{2} w^{\prime}}{\partial y^{\prime 2}}-v \frac{w^{\prime}}{K^{*}}-\frac{\sigma \beta_{0}^{2}}{\rho\left(1+m^{2}\right)}(w-m u)-K_{1}\left\{\frac{\partial^{3} w^{\prime}}{\partial t \partial y^{\prime 2}}\right\}
\end{aligned}
$$

Energy equation:

$$
\frac{\partial T^{\prime}}{\partial t^{\prime}}+v_{0} \frac{\partial T^{\prime}}{\partial y^{\prime}}=\frac{k}{\rho c p} \frac{\partial^{2} T^{\prime}}{\partial y^{\prime 2}}-\frac{1}{\rho c p} \frac{\partial q_{r}}{\partial y}+\frac{1}{\rho c p}\left(\frac{\partial u^{\prime}}{\partial y^{\prime}}\right)^{2}
$$

Concentration equation:

$$
\frac{\partial C^{\prime}}{\partial t^{\prime}}+v_{0} \frac{\partial C^{\prime}}{\partial y^{\prime}}=D \frac{\partial^{2} C^{\prime}}{\partial y^{\prime 2}}-K C^{\prime}
$$

The initial boundary conditions are:

$$
\begin{aligned}
& \left.u=0, w=0, T=T_{d}+\left(T_{0}-T_{d}\right) \varepsilon \mathrm{e}^{i \omega t}, C=C_{d}+\left(C_{0}-C_{d}\right) \varepsilon \mathrm{e}^{i \omega t} \text { at } y=0\right\} \\
& u=0, w=0, T=0, C=0 \text { at } y=d
\end{aligned}
$$

where $u$ is the velocity of the fluid in the $x$-direction and $v$ in the $y$-direction, $T$ is the temperature of the fluid, $C$ is the concentration of the fluid, $g$ is the acceleration due to gravity, $\beta^{*}$ are the kinematic viscosity, $K$ is the thermal conductivity and $C p$ is the specific heat capacity of the fluid at constant pressure. $t$ is the time, $\sigma$ is the electrical conductivity of the fluid and $\mu_{e}$ is the magnetic permeability. $T_{0}$ is the temperature of the plate and $T_{d}$ is the temperature of the fluid far away from plate. $C_{0}$ is the concentration of the plate and $C_{d}$ is the concentration of the fluid far away from the plate. $M$ is magnetic number, $K_{1}$ is viscoelastic parameter, $\varphi$ is the frequency of oscillation, $m$ is hall current parameter.

Introducing the following non-dimensional quantities, 


$$
\begin{aligned}
& w_{1}^{\prime}=\frac{U}{e} w_{1}, \partial w_{1}^{\prime}=\frac{U}{e} \mathrm{~d} w_{1}, \quad \partial^{2} w_{1}^{\prime}=\frac{U}{e} \mathrm{~d}^{2} w_{1}, \\
& y=\frac{y^{\prime}}{d}, t=\frac{t v_{0}}{v} \Rightarrow t^{\prime}=\frac{v t}{v_{0}}, \partial t^{\prime}=\frac{v}{v_{0}} \mathrm{~d} t, \\
& \theta=\frac{T^{\prime}-T_{d}^{\prime}}{T_{0}^{\prime}-T_{d}^{\prime}}, \quad M=\frac{\sigma \beta_{0}^{2} v}{\rho v_{0}^{2}}, \quad K^{*}=\frac{K_{0} v_{0}^{2}}{v^{2}} \\
& C=\frac{C^{\prime}-C_{d}^{\prime}}{C_{0}^{\prime}-C_{d}^{\prime}}, \quad G c=\frac{v \beta^{*} v\left(C_{\omega}^{\prime}-C_{\infty}^{\prime}\right)}{U v_{0}^{2}}
\end{aligned}
$$

The term $\frac{\partial q_{r}}{\partial y}$ represents the radiative heat flux. By using Rosseland approximation, the radiation heat flux $q_{r}=-\frac{4 \sigma^{*} \partial T^{4}}{3 a_{R} \partial y}$, where and Stephen and Boltzmann constants and mean absorption coefficient respectively. We assume that the temperature difference within the flow is such that may be expanded in a Taylor's series.

Hence, expanding $T^{4}$ about $T_{d}$ and neglecting higher order terms, we get

$$
T^{\prime 4}=T_{d}^{\prime 4}+4 T_{d}^{3} T^{*}=4 T_{d}^{3} T^{\prime}-3 T_{d}^{\prime 4}
$$

We assume the following solutions:

$$
\left.\begin{array}{rl}
u^{\prime} & =u_{0}^{\prime}+\varepsilon u_{1}^{\prime} \mathrm{e}^{i \omega t} \\
w^{\prime} & =w_{0}^{\prime}+\varepsilon w_{1}^{\prime} \mathrm{e}^{i \omega t} \\
\theta^{\prime} & =\theta_{0}^{\prime}+\varepsilon \theta_{1}^{\prime} \mathrm{e}^{i \omega t} \\
C^{\prime}=C_{0}^{\prime}+\varepsilon C_{1}^{\prime} \mathrm{e}^{i \omega t}
\end{array}\right\}
$$

where $L=\left(\frac{1}{K_{s}}+\frac{M}{1+m^{2}}\right), \quad J=\left(\frac{M m}{1+m^{2}}\right)$

$$
\frac{\mathrm{d}^{2} w_{0}}{\mathrm{~d} y^{2}}-\frac{\mathrm{d} w_{0}}{\mathrm{~d} y}-L w_{0}+J u_{0}=0
$$

Combining (8) and (9) using complex variable method, we have,

$$
\frac{\mathrm{d}^{2} F}{\mathrm{~d} y^{2}}-\frac{\mathrm{d} F}{\mathrm{~d} y}-F L-F J=-G c C_{0}
$$

where $F=\left(u_{0}+i w_{0}\right)$ and $i=\sqrt{-1}$

$$
\frac{\mathrm{d}^{2} F}{\mathrm{~d} y^{2}}-\frac{\mathrm{d} F}{\mathrm{~d} y}-P_{1} F=-G c C_{0}
$$

where $P_{1}=(L+J)$

$$
\Rightarrow\left(1-i \omega K_{1}\right) \frac{\mathrm{d}^{2} u_{1}}{\mathrm{~d} y^{2}}-\frac{\mathrm{d} u_{1}}{\mathrm{~d} y}-L n u_{1}+J w_{1}=-G c C_{1}^{\prime}
$$

where $L n=(L+i \omega)$ 


$$
\begin{gathered}
\Rightarrow\left(1-i \omega K_{1}\right) \frac{\mathrm{d}^{2} w_{1}}{\mathrm{~d} y^{2}}-\frac{\mathrm{d} w_{1}}{\mathrm{~d} y}-(L+i \omega) w_{1}+J u_{1}=0 \\
\Rightarrow\left(1-i \omega K_{1}\right) \frac{\mathrm{d}^{2} w_{1}}{\mathrm{~d} y^{2}}-\frac{\mathrm{d} w_{1}}{\mathrm{~d} y}-L_{0} w_{1}+J u_{1}=0
\end{gathered}
$$

Using the method of complex variable, combining (12) and (13) to have,

$$
P_{2} \frac{\mathrm{d}^{2} H}{\mathrm{~d} y^{2}}-\frac{\mathrm{d} H}{\mathrm{~d} y}-P_{3} H=-G c C_{1}
$$

where $H=\left(u_{1}+i w_{1}\right), \quad P_{2}=\left(1-i \omega K_{1}\right), \quad L=\left(L_{0}+i \omega\right)$.

The new boundary conditions are,

$$
\left.\begin{array}{l}
u_{0}^{\prime}=u_{1}^{\prime}=0, w_{0}^{\prime}=w_{1}^{\prime}=0, \theta_{0}^{\prime}=\theta_{1}^{\prime}=1, C_{0}^{\prime}=C_{1}^{\prime}=1, \text { at } y=0 \\
u_{0}^{\prime}=u_{1}^{\prime}=0, w_{0}^{\prime}=w_{1}^{\prime}=0, \theta_{0}^{\prime}=\theta_{1}^{\prime}=0, C_{0}^{\prime}=C_{1}^{\prime}=0, \text { at } y=1
\end{array}\right\}
$$

\section{Method of Solution}

To solve for mass diffusion, therefore we assume:

Concentration to be

$$
\begin{aligned}
C(y, t)= & C_{0}+C_{1} \varepsilon \exp (i \omega t) \\
C(y, t)= & A_{1} \exp \left(m_{1} y\right)+A_{2} \exp \left(m_{2} y\right) \\
& +\left(A_{3} \exp \left(m_{3} y\right)+A_{4} \exp \left(m_{4} y\right)\right) \varepsilon \exp (i \omega t)
\end{aligned}
$$

To solve for the momentum equation then

$$
\begin{gathered}
F(h)=A_{5} \exp \left(m_{5} y\right)+A_{6} \exp \left(m_{6} y\right) \\
\frac{\mathrm{d}^{2} H}{\mathrm{~d} y^{2}}-P_{4} \frac{\mathrm{d} H}{\mathrm{~d} y}-P_{5} H=0 \\
H(y)=A_{7} \exp \left(m_{7} y\right)+A_{8} \exp \left(m_{8} y\right)+D_{3} \exp \left(m_{3} y\right)+D_{4} \exp \left(m_{4} y\right)
\end{gathered}
$$

Therefore, the solution of primary velocity is assumed to be

$$
\begin{aligned}
& F\left(u_{0}+u_{1}\right)=u_{0}+u_{1} \varepsilon \mathrm{e}^{i \omega t} \\
& F\left(u_{0}+u_{1}\right) \\
& =A_{5} \exp \left(m_{5} y\right)+A_{6} \exp \left(m_{6} y\right)+D_{1} \exp \left(m_{1} y\right)+D_{2} \exp \left(m_{2} y\right) \\
& +A_{7} \exp \left(m_{7} y\right)+A_{8} \exp \left(m_{8} y\right)+D_{3} \exp \left(m_{3} y\right)+D_{4} \exp \left(m_{4} y\right) \varepsilon \mathrm{e}^{i \omega t}
\end{aligned}
$$

Then, the secondary velocity also is as follows:

$$
\begin{gathered}
H\left(w_{0}+w_{1}\right)=w_{0}+w_{1} \varepsilon \mathrm{e}^{i \omega t} \\
H\left(w_{0}+w_{1}\right) \\
=i\left\{A_{5} \exp \left(m_{5} y\right)+A_{6} \exp \left(m_{6} y\right)+D_{1} \exp \left(m_{1} y\right)+D_{2} \exp \left(m_{2} y\right)\right. \\
\left.+A_{7} \exp \left(m_{7} y\right)+A_{8} \exp \left(m_{8} y\right)+D_{3} \exp \left(m_{3} y\right)+D_{4} \exp \left(m_{4} y\right) \varepsilon \mathrm{e}^{i \omega t}\right\}
\end{gathered}
$$

From the energy equation we have,

$$
\frac{\partial T^{\prime}}{\partial t^{\prime}}+v_{0} \frac{\partial T^{\prime}}{\partial y^{\prime}}=\frac{k}{\rho c p} \frac{\partial^{2} T^{\prime}}{\partial y^{\prime 2}}-\frac{1}{\rho c p} \frac{\partial q_{r}}{\partial y}+\frac{1}{\rho c p}\left(\frac{\partial u^{\prime}}{\partial y^{\prime}}\right)^{2}
$$


Substituting the value of $\partial T^{\prime}$ yields

$$
\frac{\partial \theta}{\partial t}+\frac{\partial \theta}{\partial y}=\frac{1}{\operatorname{Pr}} \frac{\partial^{2} \theta}{\partial y^{2}}+\frac{4 R \partial^{2} \theta}{3 \operatorname{Pr} \partial y^{2}}+E c\left(\frac{\partial u^{\prime}}{\partial y^{\prime}}\right)^{2}
$$

where $E c=\frac{v}{\rho C_{p}\left(T_{0}^{\prime}-T_{d}^{\prime}\right)}$

$$
\frac{\partial \theta}{\partial t}+\frac{\partial \theta}{\partial y}=\frac{E}{\operatorname{Pr}} \frac{\partial^{2} \theta}{\partial y^{2}}+E c\left(\frac{\partial u^{\prime}}{\partial y^{\prime}}\right)^{2}
$$

where $E=\left(1+\frac{4 R}{3}\right)$

$$
\frac{\partial \theta}{\partial t}+\frac{\partial \theta}{\partial y}=\frac{E}{\operatorname{Pr}} \frac{\partial^{2} \theta}{\partial y^{2}}+E c\left(\frac{\partial u^{\prime}}{\partial y^{\prime}}\right)^{2}
$$

We also assumed solution to be

$$
\left.\begin{array}{l}
\theta=\theta_{0}+\theta_{1} \varepsilon \mathrm{e}^{i \omega t} \\
u=u_{0}+u_{1} \varepsilon \mathrm{e}^{i \omega t}
\end{array}\right\}
$$

Therefore, the solution of the energy equation is as follows:

$$
\begin{aligned}
\theta= & \left\{A+B \exp \left(\operatorname{Pr} E_{2} y\right)+D_{11} \exp \left(2 m_{5} y\right)+D_{12} \exp \left(2 m_{6} y\right)\right. \\
& +D_{13} \exp \left(2 m_{1} y\right)+D_{14} \exp \left(2 m_{2} y\right)+D_{15} \exp \left(m_{5}+m_{6}\right) y \\
& +D_{16} \exp \left(m_{5}+m_{1}\right) y+D_{17} \exp \left(m_{5}+m_{2}\right) y+D_{18} \exp \left(m_{6}+m_{1}\right) y \\
& \left.+D_{19} \exp \left(m_{6}+m_{2}\right) y+D_{20} \exp \left(m_{1}+m_{2}\right) y\right\} \\
& +\left\{A_{9} \exp \left(m_{9} y\right)+A_{10} \exp \left(m_{10} y\right)+D_{21} \exp \left(m_{5}+m_{7}\right) y\right. \\
& +D_{22} \exp \left(m_{5}+m_{8}\right) y+D_{23} \exp \left(m_{5}+m_{3}\right) y+D_{24} \exp \left(m_{5}+m_{4}\right) y \\
& +D_{25} \exp \left(m_{6}+m_{7}\right) y+D_{26} \exp \left(m_{6}+m_{8}\right) y+D_{27} \exp \left(m_{6}+m_{3}\right) y \\
& +D_{28} \exp \left(m_{6}+m_{4}\right) y+D_{29} \exp \left(m_{1}+m_{7}\right) y+D_{30} \exp \left(m_{1}+m_{8}\right) y \\
& +D_{31} \exp \left(m_{1}+m_{3}\right) y+D_{32} \exp \left(m_{1}+m_{4}\right) y+D_{33} \exp \left(m_{2}+m_{7}\right) y \\
& +D_{34} \exp \left(m_{2}+m_{8}\right) y+D_{35} \exp \left(m_{2}+m_{3}\right) y \\
& \left.+D_{36} \exp \left(m_{2}+m_{4}\right) y\right\} \varepsilon \exp (i \omega t)
\end{aligned}
$$

\section{Results and Discussion}

\subsection{Velocity Profiles}

Figures 1-10 represent velocity profile for the flow.

\subsection{Concentration Profiles}

Figure 11 and Figure 12 represent concentration profiles for the flow.

\subsection{Temperature Profiles}

Figure 13 and Figure 14 represent temperature profiles for different values of Prandtle number $(P r)$ and radiation parameter $(R)$ of the flow. 


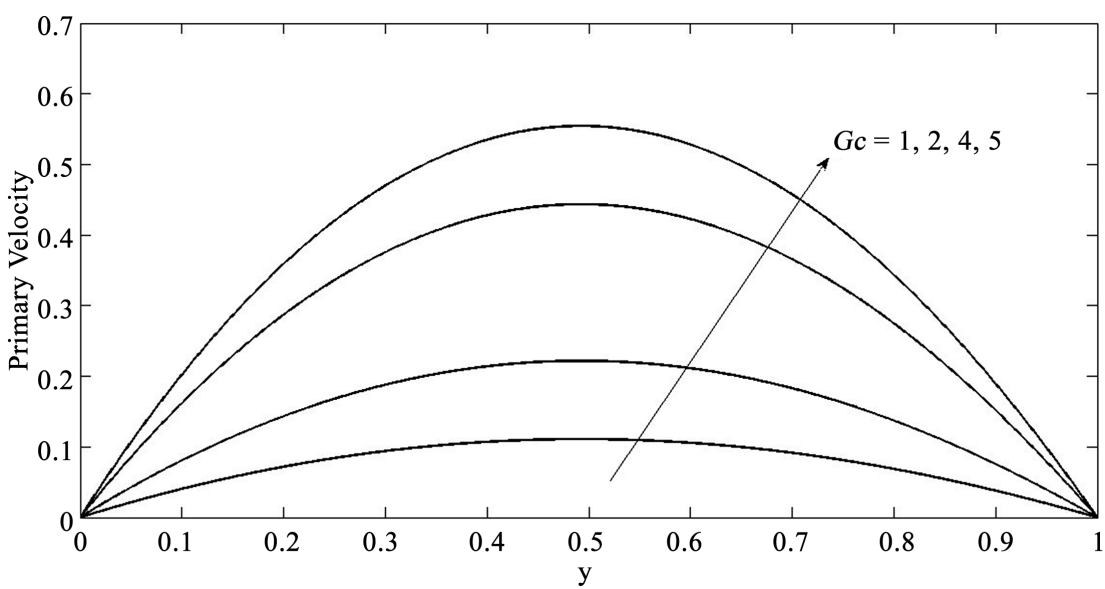

Figure 1. Primary velocity profiles for different values of mass Grashof number $(G c)$. This indicates that the primary velocity attends its peak at $y=0.5$, in the same vein primary velocity increase with increase in the values of $G c$.

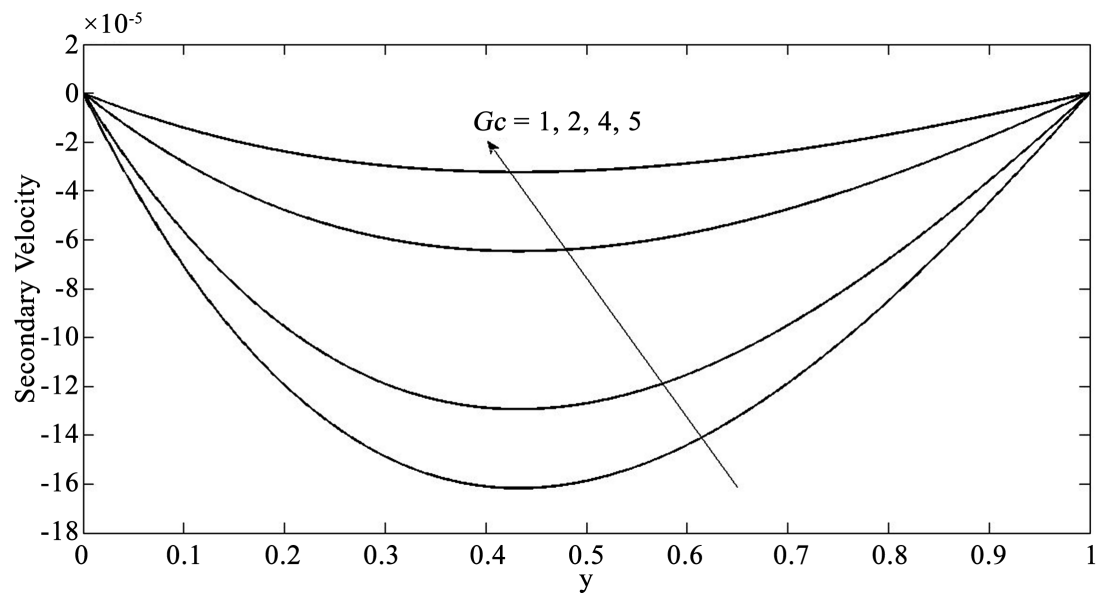

Figure 2. Secondary velocity profiles for different values of Thermal Grashof number $(G c)$. The least value was attended at $y=0.45$, consequently the velocity of the fluid increase with increase in values of $G c$.

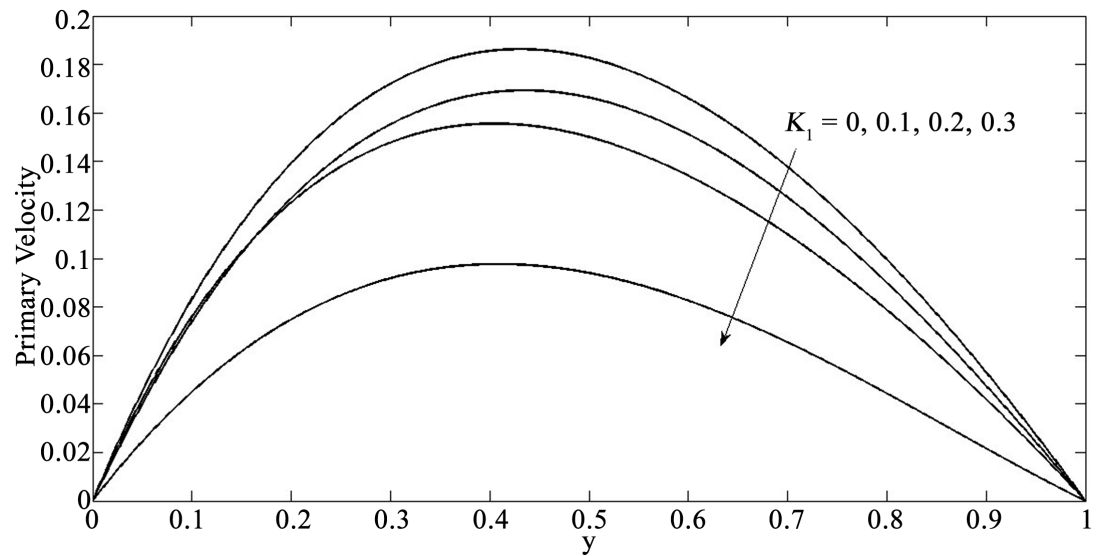

Figure 3. Primary velocity profiles for different values of viscoelastic parameter $\left(K_{1}\right)$. In this flow primary velocity decrease with increasing values of $K_{1}$. This is in agreement with general belief that viscoelastic causes drag in a flow of a fluid. 


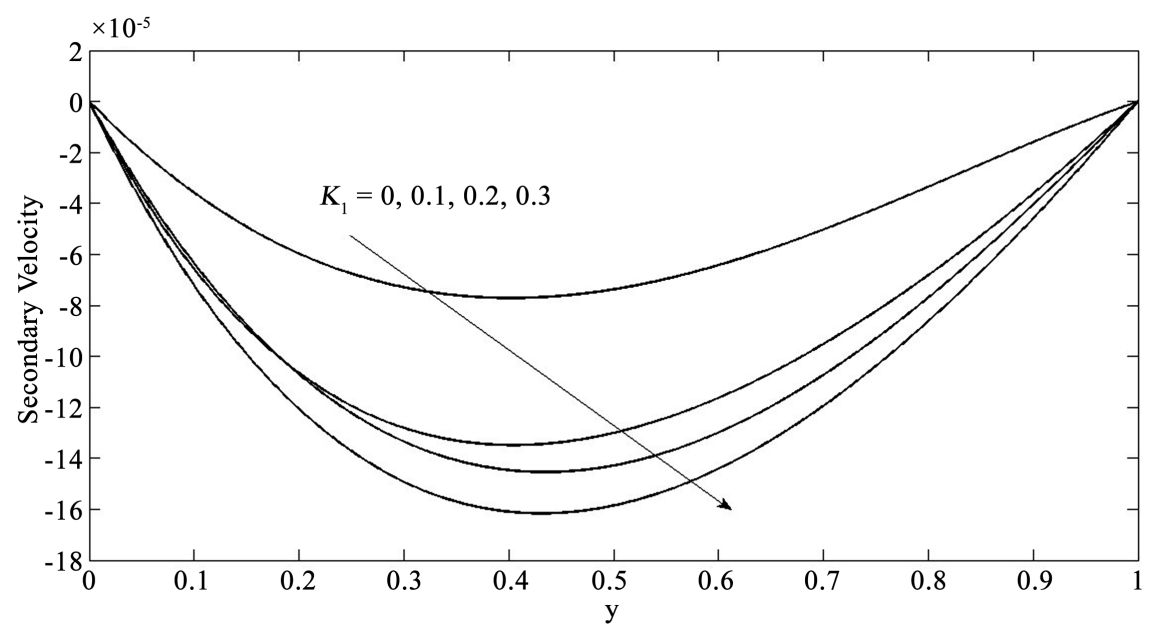

Figure 4. Secondary velocity profiles for different values of $K_{1}$, this demonstrated the same behavior as in primary velocity.

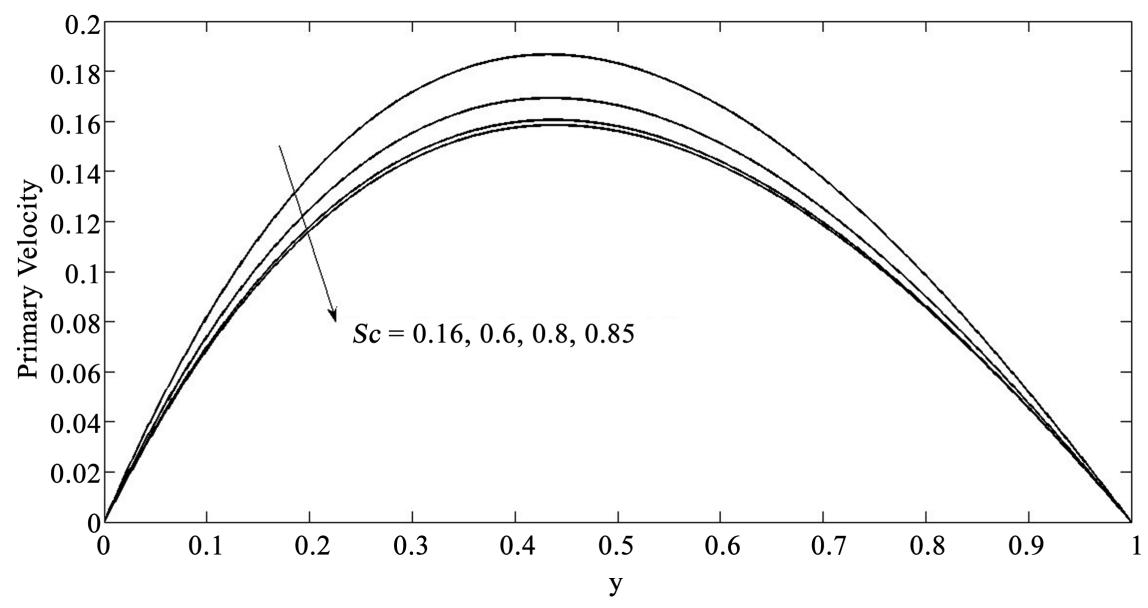

Figure 5. Primary velocity profiles for different values of Schmidt number (Sc). As $S c$ increases the primary velocity decreases.

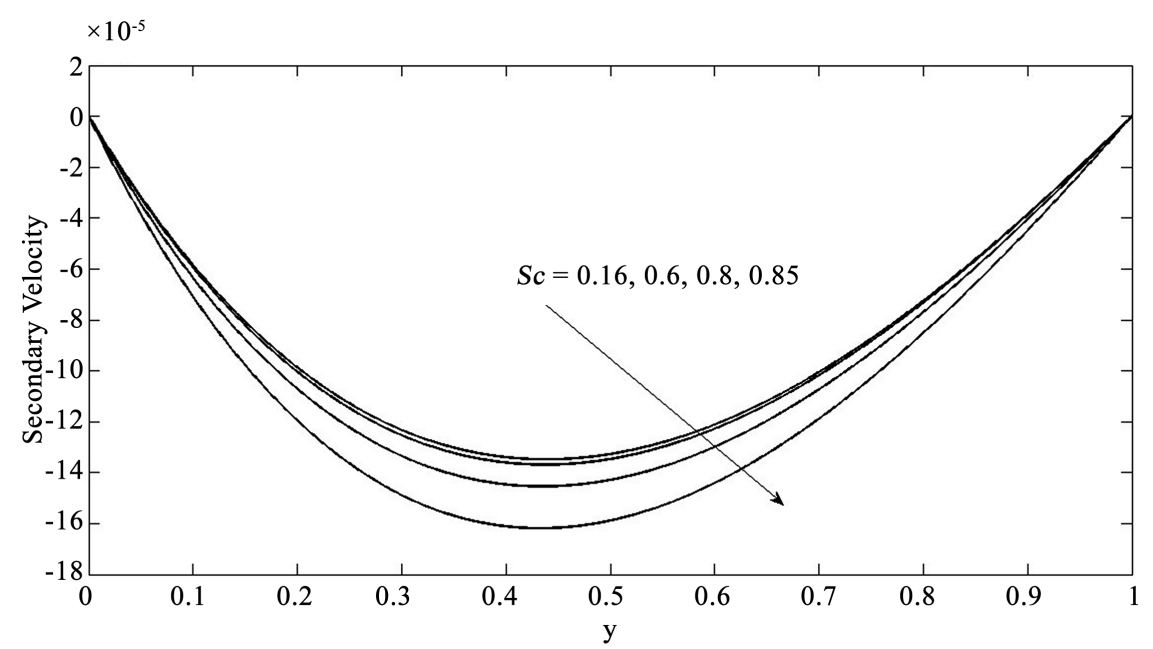

Figure 6. Secondary velocity profiles for different values of $S c$ indicated the same trend as in the case of primary velocity. 


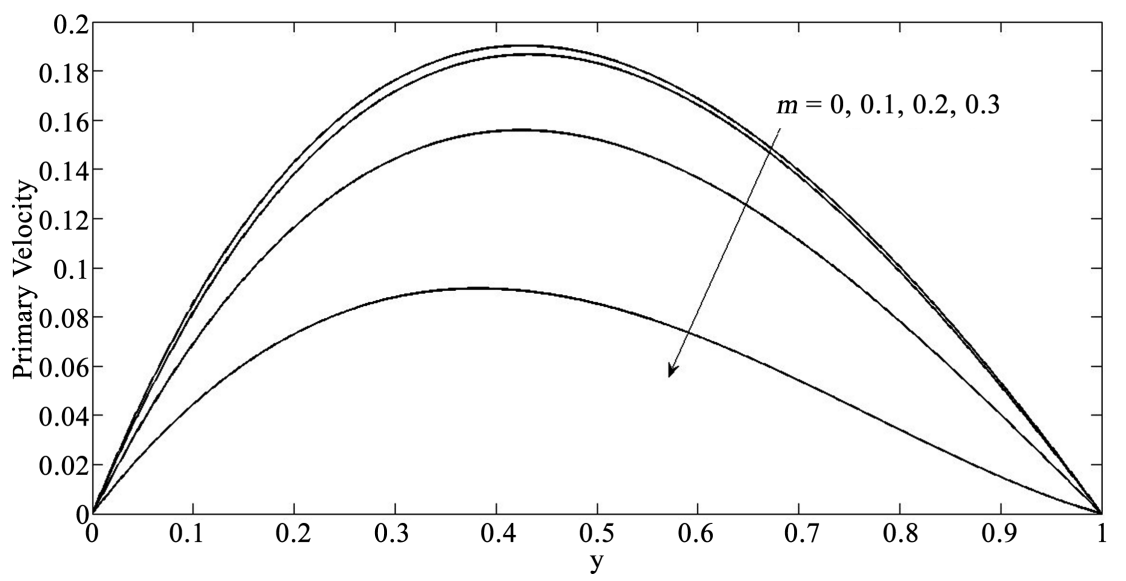

Figure 7. Primary velocity profiles for different values of hall current parameter $(m)$. When $m=0$ the velocity reached its maximum at 0.19 units, $y=0.45$, as the values of $m$ increases the velocity reduced to as low as 0.08 units. This means that hall current has adverse effects on flow of this fluid.

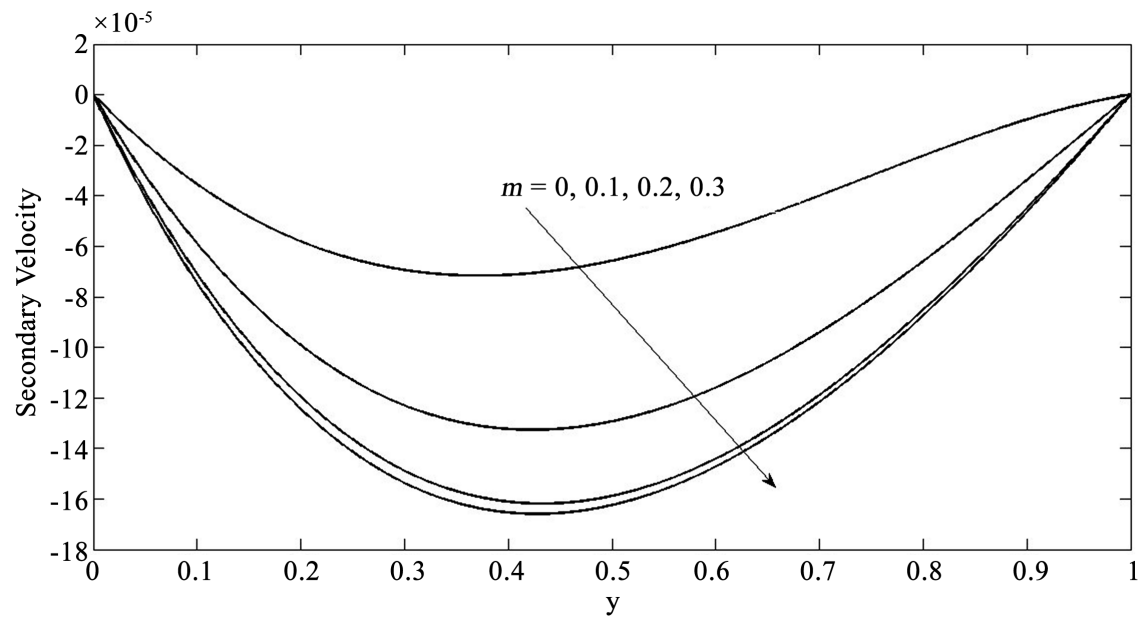

Figure 8. Secondary velocity profiles for different values of $m$. when $m=0$ the flow was low, it became lowest as the values of $m$ increased.

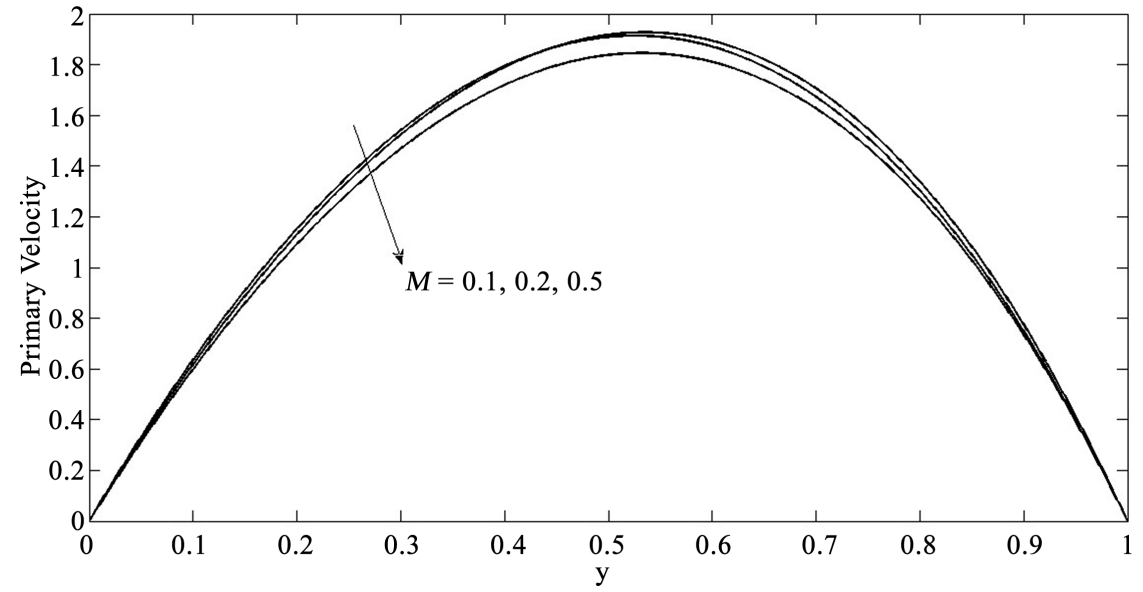

Figure 9. Primary velocity profiles for different values of magnetic field $(M)$. The effects of $M$ in this flow are insignificant, even though it reduces the flow of the fluid. 


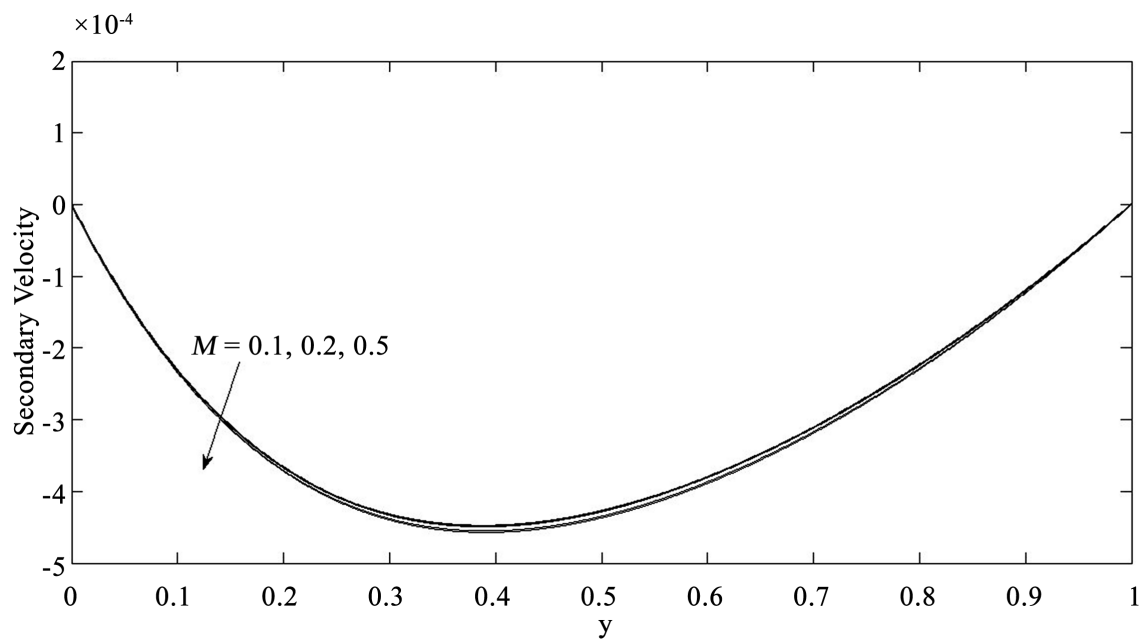

Figure 10. Primary velocity profiles for different values of magnetic field ( $M$ ).

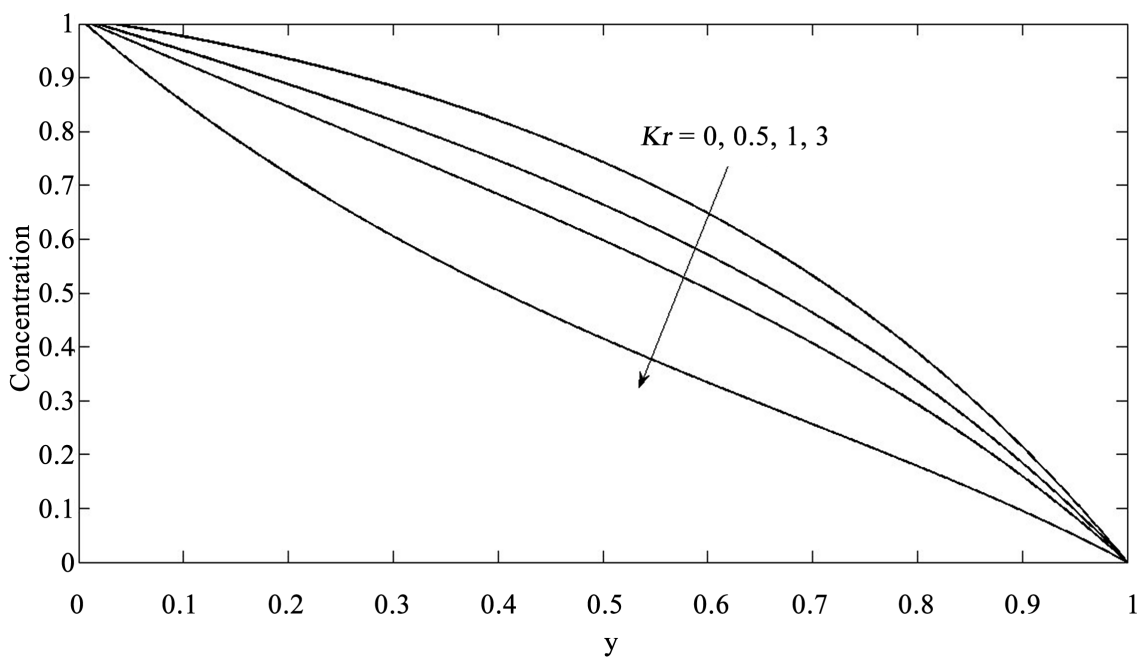

Figure 11. Concentration profiles for different values of chemical reaction parameter $(K r)$.

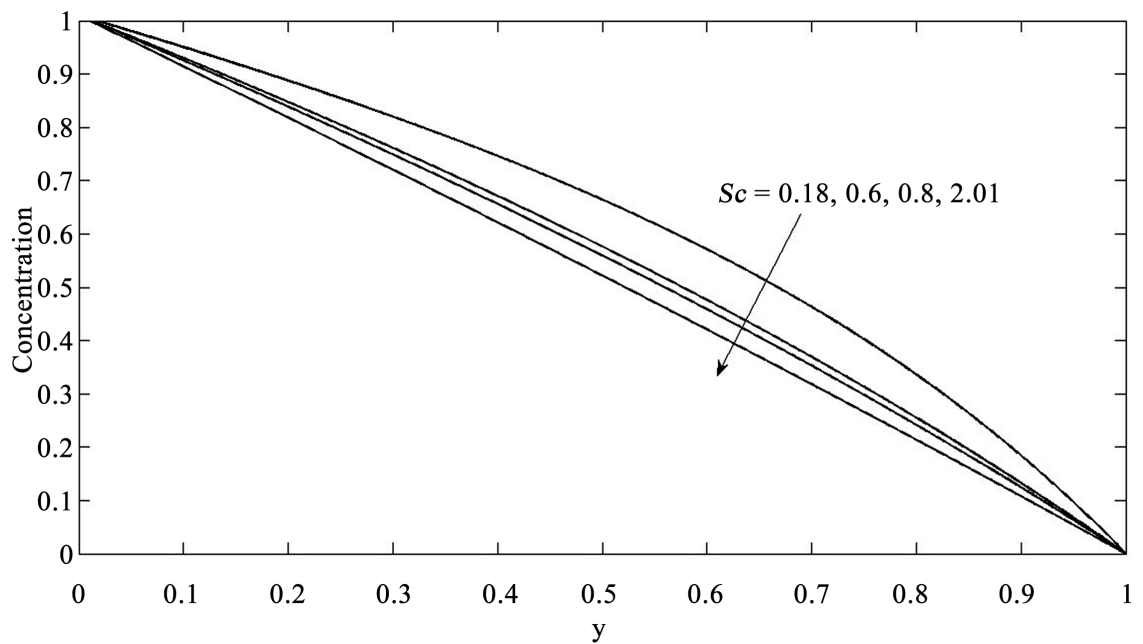

Figure 12. Concentration profiles for different values of Schmidt number $(S c)$. 


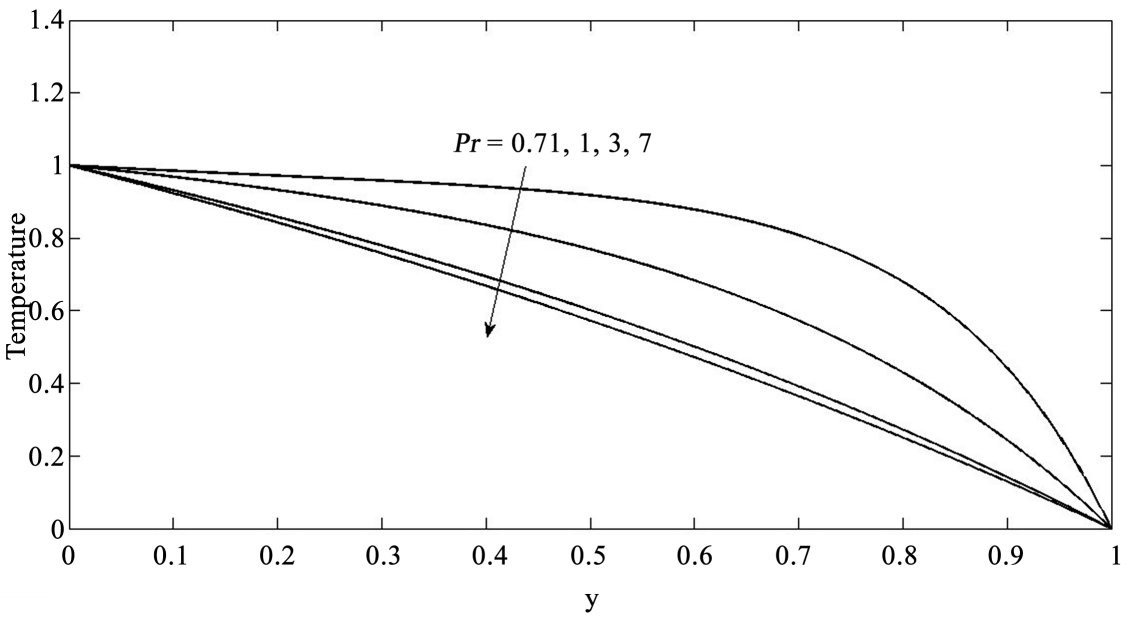

Figure 13. Temperature profiles for different values of Prantle number $(P r)$. The graph demonstrated uniformity where $\operatorname{Pr}=0.71$ and 1.0 , while the last two values did not show significant effect on temperature.

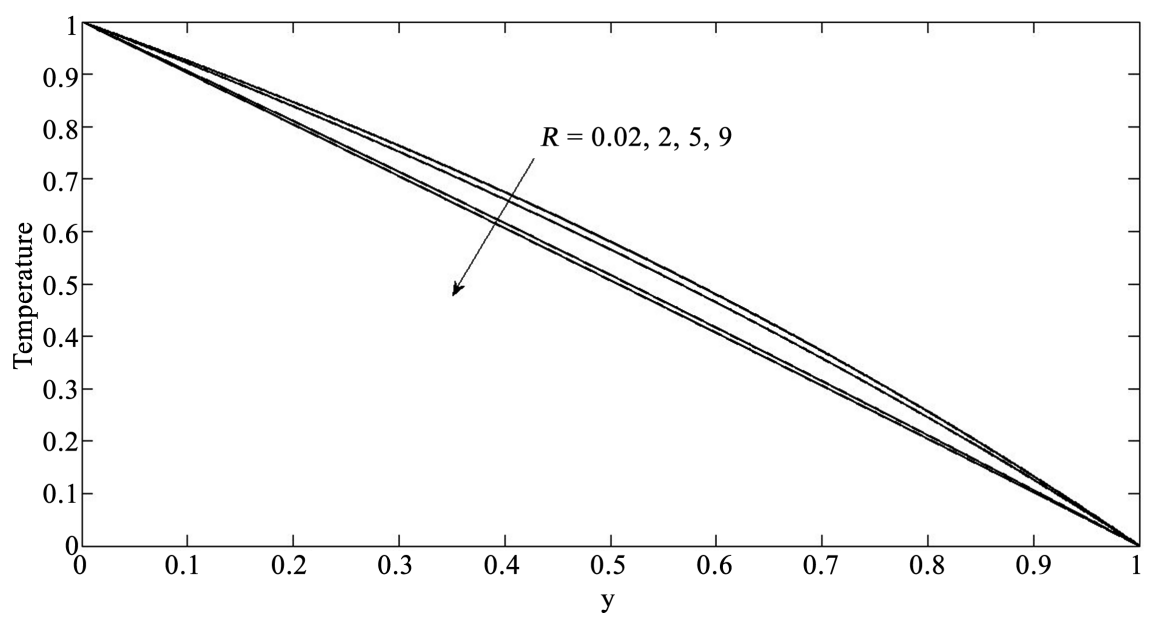

Figure 14. Temperature profiles for different values of radiation parameter $(R)$. The effects of radiation parameter are insignificant as demonstrated in the figure above.

\section{Conclusion}

Investigation of effects of some physical parameters and hall current on MHD fluid flow with heat flux over a porous medium is studied by transforming the governing partial differential equations into ordinary differential equations which are then solved using perturbation techniques. The result of the flow variables indicates that the fluid temperature is reduced by increasing Prandtl number $(P r)$ and radiation parameter $(R)$. Concentration is reduced with increase in Schmidt number $(S c)$ and chemical reaction parameter $(K)$. The primary velocity decrease with increasing prandtl $(P r)$, radiation parameter and hall-current while the opposite trend is observed in secondary velocity. The primary velocity increases with increase in mass Grashoof number $(G r)$ and thermal Grasshoof number $(G c)$ also the reverse is the case in secondary velocity. The primary velocity decreases with increase in $M, s$ and $S c$. 


\section{Conflicts of Interest}

The authors declare no conflicts of interest regarding the publication of this paper.

\section{References}

[1] Alam, M.S., Rahaman, M.M. and Satar, M.A. (2008) Effects of Variable Suction and Thermophoresis on Steady MHD Combined Free-Forced Convective Heat and Mass Transfer Flow over a Semi Infinite Permeable Inclined Plate in the Presence of Thermal Radiation. International Journal of Thermal Science, 47, 758-765. https://doi.org/10.1016/j.ijthermalsci.2007.06.006

[2] Veera Krishna, M. and Sujatha, P. (2011) MHD Free and Forced Convection Flow of Newtonian Fluid through a Porous Medium in an Infinite Vertical Plate in Presence of Thermal Radiation Heat Transfer and Surface Temperature Oscillation. International Journal of Physics and Mathematical Science, 1, 33-52.

[3] Saleh, M.A., Mohammed, A.A.B. and Mahmoud, S.E. (2010) Heat and Mass Transfer in MHD Viscous Elastic Fluid Flow through a Porous Medium over a Stretching Sheet with Chemical Reaction, Journal of Applied Mathematics, 1, 446-455. https://doi.org/10.4236/am.2010.16059

[4] Malraju, C.R., Narravula, A.R. and Sibyale, V.K. (2011) Hallcurrent Effects on Unsteady Magnetohydrodynamics Flow between Stretching Sheet and an Oscillating Porous Upper Parallel Plate with Constant Suction. Thermal Science, 15, 527-536. https://doi.org/10.2298/TSCI1102527C

[5] Kafousias, N.G. and Raptis, A.A. (1981) Mass Transfer and Free Convection Effects on the Flow Past an Accelerated Vertical Plate with Variable Suction or Injection. International Journal of Engineering Science, 26, 11-22.

[6] Uwanta, I.J. and Ahmed, A. (2012) Radiation Effects on Heat and Mass Transfer over a Vertical Plate with Newtonian Fluid. International Journal of Engineering Research and Technology (IJERT), 1, 1-15.

[7] Ahmed, A., Uwanta, I.J. and Sarki, M.N. (2015) Hall Current Effects on Unsteady MHD Fluid Flow with Radiative Heat Flux and Heat Source over a Porous Medium. British Journal of Mathematics and Computer Science, 6, 233-246. https://doi.org/10.9734/BJMCS/2015/13849

[8] Anwerbeg, O. and Ghosh, S.K. (2010) Analytical Study of Magneto Hydrodynamic Radiation-Convection with Surface Temperature Oscillation and Secondary Flow Effects. International Journal of Applied Mathematics and Mechanics, 6, 1-22.

[9] Gupta, A.S. (1975) Hydro Magnetic Flow past a Porous Flat Plate with Hall Effects. Acta Mechanica, 22, 281-287. https://doi.org/10.1007/BF01170681

[10] Singh, A.K. and Singh, N.P. (2003) Heat and Mass Transfer in MHD Flow of a Viscous Fluid Past a Vertical Plate under Oscillatory Suction Velocity. Indian Journal of Pure and Applied Mathematics, 34, 429-442.

[11] Ferdows, M., Kainoota, K. and Crepeau, J.C. (2008) MHD Free Convection and Mass Transfer Flow in a Porous Media with Simultaneous Rotating Fluid. International Journal of Dynamics of Fluids, 4, 69-82.

[12] Ramana Murthy, M.V., et al. (2007) MHD Unsteady Free Convective Walter's Memory Flow with Constant Suction and Heat Sink. AAPN Journal of Engineering and Applied Science, 2, 12-16.

[13] Ahmed, A., Sarki, M.N. and Ahmad, M. (2014) Radiation Effects on Heat and Mass Transfer over an Exponentially Accelerated Infinite Vertical Plate with Chemical 
Reaction. Proceeding of the International Multiconference of Engineers and Computer Scientists, Vol. II, Hong Kong, 12-14 March 2014, 914-918.

[14] Sreedevi, R.R., Parasoda, D.R.V. and Chamkha, A.J. (2016) Combined Influence of Radiation Absorption and Hall Current Effects on MHD Double Diffusive Free Convective Flow past a Stretching Sheet. Ain Shams Engineering Journal, 7, 383-397. https://doi.org/10.1016/j.asej.2015.11.024

[15] Babu, D. and Raju, G.S.S. (2017) Hall Current Effects on MHD Free Convective Heat and Mass Transfer Flow past an Oscillating Vertical Porous Plate in the Presence of Radiation, Thermal and Mass Diffusion with Chemical Reaction. International Journal of Mathematics and Computer Application Research (IJMCAR), 7, 1-22.

[16] Rajput, U.S. and Kumar, G. (2017) Effects of Radiation, Chemical Reaction and Porosity of the Medium on MHD Flow past an Oscillating Plate with Heat and Mass Transfer. Aryabhatta Journal of Mathematics and Informatics, 9, 230-241.

[17] Changal, M.R., Narravula, A.R. and Silbiya, V.K. (2011) Hall Current Effects on Unsteady Magnetohydrodynamics Flow between Stretching Sheet and an Oscillating Porous Upper Parallel Plate with Constant Suction. Thermal Science, 15, 527-536. https://doi.org/10.2298/TSCI1102527C

[18] Kumar, R. and Chand, K. (2011) Effect of Slip Conditions and Hall Current on Unsteady MHD Flow of a Viscoelastic Fluid Past on Infinite Vertical Porous Plate through Porous Medium. International Journal of Engineering Science and Technology, 3, 3124-3133. 